\title{
Investigation on Electromagnetic Models of High-Speed Solenoid Valve for Common Rail Injector
}

\author{
Jianhui Zhao, ${ }^{1}$ Liyun Fan, ${ }^{1}$ Peng Liu, ${ }^{1}$ Leonid Grekhov, ${ }^{2}$ Xiuzhen $M a,{ }^{1}$ and Enzhe Song ${ }^{1}$ \\ ${ }^{1}$ College of Power and Energy Engineering, Harbin Engineering University, Harbin 150001, China \\ ${ }^{2}$ College of Power Engineering, Bauman State Technical University, Moscow 115569, Russia \\ Correspondence should be addressed to Liyun Fan; fanly_01@163.com
}

Received 26 December 2016; Revised 10 April 2017; Accepted 20 April 2017; Published 12 June 2017

Academic Editor: Francesco Braghin

Copyright (c) 2017 Jianhui Zhao et al. This is an open access article distributed under the Creative Commons Attribution License, which permits unrestricted use, distribution, and reproduction in any medium, provided the original work is properly cited.

\begin{abstract}
A novel formula easily applied with high precision is proposed in this paper to fit the $B-H$ curve of soft magnetic materials, and it is validated by comparison with predicted and experimental results. It can accurately describe the nonlinear magnetization process and magnetic saturation characteristics of soft magnetic materials. Based on the electromagnetic transient coupling principle, an electromagnetic mathematical model of a high-speed solenoid valve (HSV) is developed in Fortran language that takes the saturation phenomena of the electromagnetic force into consideration. The accuracy of the model is validated by the comparison of the simulated and experimental static electromagnetic forces. Through experiment, it is concluded that the increase of the drive current is conducive to improving the electromagnetic energy conversion efficiency of the HSV at a low drive current, but it has little effect at a high drive current. Through simulation, it is discovered that the electromagnetic energy conversion characteristics of the HSV are affected by the drive current and the total reluctance, consisting of the gap reluctance and the reluctance of the iron core and armature soft magnetic materials. These two influence factors, within the scope of the different drive currents, have different contribution rates to the electromagnetic energy conversion efficiency.
\end{abstract}

\section{Introduction}

The fuel system has a vital impact on the overall performance of a diesel engine, and a high-pressure common rail system enables the cyclic injection quantity, injection timing, and fuel injection law to be controlled exactly and flexibly. Therefore, a diesel engine that is equipped with a highpressure common rail system has the potential to achieve the optimized design goals of high-efficiency combustion and ultralow emission [1-5]. The common rail injector is one of the high-pressure common rail system's critical components, with a direct influence on the cycle fuel injection quantity fluctuation, gas-liquid two-phase flow characteristics of highpressure fuel in the nozzle, fuel atomization characteristics in the cylinder, and air-fuel mixture quality [6-9]. The common rail injector is a complex, nonlinear, and multidimensional system that couples electromagnetic, mechanical, and hydraulic phenomena. To study the dynamic characteristics of the common rail injector in detail and optimize it, the simulation analysis of its dynamic performance is an effective method. A simulation model of the HSV that has high accuracy is thus indispensable.

The dynamic response characteristic is an important evaluation index of the HSV in the fuel injector. This is because a fast response speed of the HSV is beneficial to achieve multiple injections and more precise control of the fuel injection timing of the high-pressure common rail system. The dynamic response of the HSV is determined by both its electromagnetic force characteristic and moving part quality, but the former has a more significant impact. Only when the maximum static electromagnetic force of the HSV meets its requirements can the response time requirements of the opening and closing phases of the HSV be met by using the drive circuit. Hence, research on the electromagnetic force is of great importance for the design of the HSV.

In research on the static electromagnetic force of the HSV, most scholars used the finite element method (FEM). Liu et al. [10] studied the influence rules of the main 
structure parameters and the interaction between them on the electromagnetic force using a combination of response surface design and FEM. Sun et al. [11] studied the influence rules of the iron core length, the cross-sectional areas of the main and side poles, the coil turns, and the air gap width on the electromagnetic force of an E-shaped HSV for an electronic unit pump by FEM. It was discovered that the drive current significantly impacts the electromagnetic energy conversion, as increasing the drive current unnecessarily does not improve the electromagnetic force of the HSV but only increases the power consumption and thus reduces the electromagnetic energy conversion efficiency. Cheng et al. [12] studied using FEM the flux density distribution of an HSV whose iron core is made of a nano-based soft magnetic material. It was discovered that solenoid valves with different soft magnetic materials have different characteristics of electromagnetic force. Miller et al. [13], Shin et al. [14], and Bianchi et al. [15] carried out optimization research on the static electromagnetic force of an HSV to obtain the optimal structure parameters by FEM. However, because of the long solution time and high requirements for computer performance of FEM, the efficiency of research on the optimization and analysis of the structural parameters is low, especially when carrying out an iterative structural analysis for the detailed parameter design of HSV. Therefore, the development of a static electromagnetic mathematical model for an HSV that can be applied conveniently with high accuracy has become an important research direction.

Elmer and Gentle [16] put forward a relatively simple mathematical model of a proportional solenoid valve. The model simplified the electromagnetic process calculation of the solenoid valve as the calculation of an $\mathrm{RL}$ equivalent circuit. Ma et al. [17], Shamdani et al. [18], and Bianchi et al. [19] determined the influencing relation of drive current and air gap on electromagnetic force by a polynomial fit technique based on test data of the static electromagnetic force. Wang et al. [20] and Chung et al. [21] considered the magnetic saturation characteristics of an HSV by adjusting the coefficient of the fitting formula to limit the maximum electromagnetic force of the valve. Huber and Ulbrich [22] and Chung et al. [23] developed a dynamic characteristic mathematical model for the common rail injector. Its electromagnetic submodel was built based on a map chart consisting of the electromagnetic force, drive current, and air gap. However, the HSV electromagnetic characteristics are not only a simple function relationship between the electromagnetic force and the drive current and air gap. In the high-pressure common rail system, the nonlinear transient coupling characteristics of the electromagnetic, mechanical, and hydraulic systems determine the electromagnetic characteristic transformation of the HSV. Therefore, this method meets the demands of engineering application, but it cannot reveal the intrinsic electromagnetic properties of the HSV, both the static electromagnetic characteristics and the dynamic electromagnetic characteristics.

Most of the literature on the HSV tends to ignore the influence of the soft magnetic material reluctance in developing the electromagnetic model of the solenoid valve. Topçu et al. [24], Jin et al. [25], Naseradinmousavi and
Nataraj [26], and Mehmood et al. [27] considered that the reluctance of the iron core magnetic material is much less than that of the air gap; therefore, the nonlinear magnetization process and magnetic saturation characteristics of the iron core magnetic material can be ignored. Sefkat [28] assumed that the magnetic field is not saturated in the whole working process of the solenoid valve, and the magnetic induction intensity always linearly increases with the increasing magnetic field intensity; that is to say, the saturation phenomenon of the electromagnetic force did not happen for their model. However, Wang et al. [29] and Sun et al. [11] discovered that a saturation phenomenon of the electromagnetic force for the solenoid valve existed in their experimental research, and the magnetic saturation of the HSV soft magnetic materials was the main cause of this. In the actual control process of the HSV, to improve the opening speed, a high voltage was loaded on the HSV to quickly obtain a large current. In this way, it is easy to achieve magnetic saturation for the solenoid valve. When the solenoid valve reached magnetic saturation, with the increase in the drive current, the electromagnetic force of the HSV will increase very slowly; therefore, the economy of the HSV decreased. Simply dealing with the magnetic saturation characteristic or taking no account of it will lead to obvious calculation errors in determining the electromagnetic force characteristics. Therefore, it is necessary to take the nonlinear magnetization characteristics and magnetic saturation characteristics of the solenoid valve magnetic materials into account upon building a mathematical model of an HSV.

Coppo et al. [30] established a complete mathematical model for a common rail injector. For its electromagnetic submodels, the magnetization process of the solenoid valve was divided into a linear magnetization phase and a saturation magnetization phase, which are described by simple piecewise lines. In the mathematical model of an HSV developed by Liu et al. [31], the reluctance of the soft magnetic materials was included to consider the nonlinear magnetization characteristic, but the most important $B-H$ magnetizing curve was determined based on $B-H$ experimental data by a trial and error method for calculating the reluctance of soft magnetic materials. The mathematical models of a solenoid valve developed by Jin et al. [25] and Vu et al. [32] considered the magnetic saturation characteristics, edge effect of the air gap, and flux leakage, but a detailed description of the $B-H$ magnetizing curve that determines the reluctance of the soft magnetic materials and calculates their transient permeability was not provided.

It can be found from the above references that a certain research insufficiency on the electromagnetic mathematical model of the HSV still exists. Some electromagnetic mathematical models use an electromagnetic force fitting formula based on experimental data, some models do not consider the reluctance of the soft magnetic materials, and some only took segmental magnetic properties of the magnetic materials into account. Therefore, to provide more valuable information on the electromagnetic energy conversion of the HSV and more deeply understand the solenoid valve's electromagnetic conversion process, more detailed research on the electromagnetic mathematical model of the HSV 


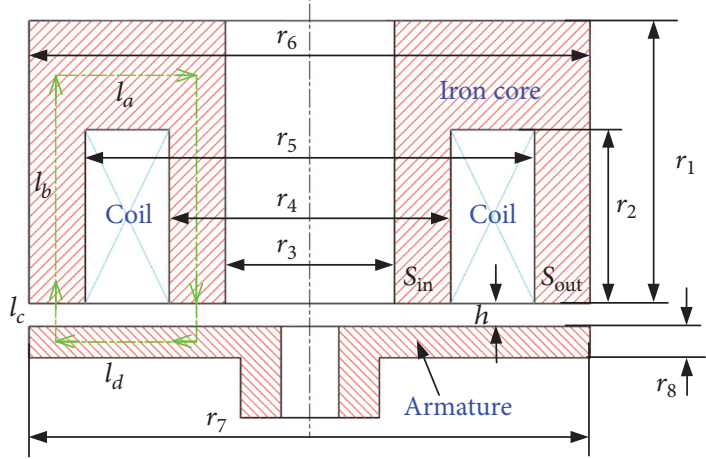

FIGURE 1: The structure schematic of HSV.

has been carried out in this paper. The resulting model considers the nonlinear magnetization characteristics and magnetic saturation characteristics of the HSV. Based on this mathematical model, research on the electromagnetic force characteristics of the common rail injector HSV and its key influence factors can follow. It provides a certain theoretical basis and design tools for the design of HSVs.

\section{The Electromagnetic Model of HSV}

2.1. Magnetic Circuit Model of HSV. Figure 1 shows the structure schematic of a HSV. It mainly consists of an iron core, coil, and armature. To obtain a strong electromagnetic force, a soft magnetic material with a high saturation induction density and low remanence is often applied for the iron core and armature.

For the HSV, the functional relationship between the total magnetic flux and the total reluctance of the magnetic circuit is described as

$$
\Phi=\frac{N \cdot I}{R_{\text {total }}}
$$

where $\Phi$ is the total magnetic flux; $N$ is the coil turns; $I$ is the current; $R_{\text {total }}$ is the total reluctance of magnetic circuit.

To accurately describe the nonlinear magnetization and magnetic saturation of the HSV, the mathematical model should take the reluctance of the iron core and armature soft magnetic materials into consideration. Due to the different equivalent cross-sectional areas of the main and side poles under normal circumstances, the gap reluctances between the main pole, side pole, and armature are described separately in the mathematical model, and the fringe effects of the air gap and the leakage flux are ignored. Based on the equivalent magnetic circuit shown in Figure 2, the total reluctance of the HSV is expressed as

$$
R_{\text {total }}=R_{\text {gap } 1}+R_{\text {gap } 2}+R_{\text {arm }}+R_{\text {iron }}
$$

where $R_{\text {gap } 1}$ is the gap reluctance between main pole and armature; $R_{\text {gap2 } 2}$ is the gap reluctance between side pole and armature; $R_{\mathrm{arm}}$ is the reluctance of armature; $R_{\mathrm{iron}}$ is

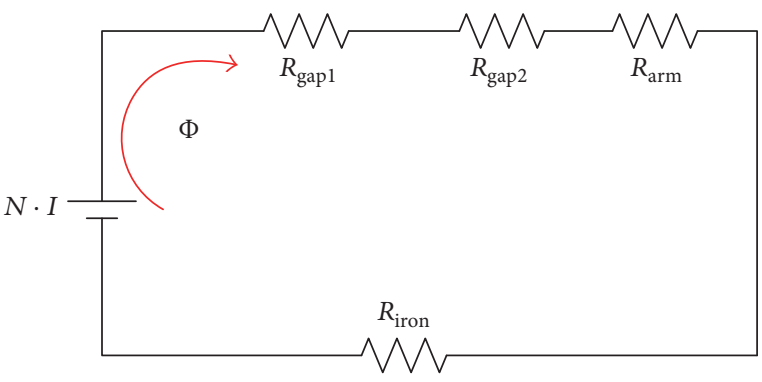

FIgURE 2: Equivalent magnetic circuit of HSV.

the reluctance of iron core. Corresponding reluctances are described as

$$
\begin{aligned}
& R_{\text {gap } 1}=\frac{h}{\mu_{0} \cdot S_{\text {in }}}, \\
& R_{\text {gap } 2}=\frac{h}{\mu_{0} \cdot S_{\text {out }}}, \\
& R_{\text {arm }}=\frac{r_{8}}{2 \cdot \mu \cdot S_{\text {in }}}+\frac{r_{8}}{2 \cdot \mu \cdot S_{\text {out }}}+\frac{l_{d}}{\mu \cdot S^{\prime \prime}}, \\
& R_{\text {iron }}=\frac{l_{b}}{\mu \cdot S_{\text {in }}}+\frac{l_{b}}{\mu \cdot S_{\text {out }}}+\frac{l_{a}}{\mu \cdot S^{\prime}},
\end{aligned}
$$

where $S_{\text {in }}$ is the equivalent cross-sectional area of the HSV main pole; $S_{\text {out }}$ is the equivalent cross-sectional area of the $\mathrm{HSV}$ side pole; $h$ is the air gap of the HSV; $l_{b}$ is the equivalent magnetic circuit length of the iron core in the axial direction; $l_{a}$ is the equivalent magnetic circuit length of the iron core in the radial direction; $l_{d}$ is the equivalent magnetic circuit length of the armature in the radial direction; $\mu$ is the permeability of the soft magnetic material, depending on the $B-H$ fundamental magnetizing curve; $S_{11}$ is the equivalent magnetic flux area of the iron core in the radial direction; and $S_{22}$ is the equivalent magnetic flux area of the armature in the radial direction. Each equivalent magnetic circuit length is expressed as

$$
\begin{aligned}
l_{b} & =\frac{r_{1}+r_{2}}{2}, \\
l_{a} & =\frac{r_{5}+r_{6}-r_{3}-r_{4}}{4}, \\
l_{d} & =l_{b}, \\
S_{11} & =\pi \cdot\left(r_{1}-r_{2}\right) \cdot \frac{r_{5}+r_{6}+r_{3}+r_{4}}{4}, \\
S_{22} & =\pi \cdot r_{8} \cdot \frac{r_{5}+r_{6}+r_{3}+r_{4}}{4}, \\
S_{\text {in }} & =\frac{\pi}{4} \cdot\left(r_{4}^{2}-r_{3}^{2}\right), \\
S_{\text {out }} & =\frac{\pi}{4} \cdot\left(r_{6}^{2}-r_{5}^{2}\right) .
\end{aligned}
$$


Finally, the electromagnetic force is computed by

$$
F_{\text {mag }}=\frac{1}{2} \frac{\phi^{2}}{\mu_{0} \cdot S_{\text {in }}}+\frac{1}{2} \frac{\phi^{2}}{\mu_{0} \cdot S_{\text {out }}} .
$$

2.2. B-H Magnetization Curve Model. The permeability of the soft magnetic material shows its influence by two aspects in the electromagnetic mathematical model of the HSV. On the one hand, the reluctance of the soft magnetic material has an effect on the electromagnetic conversion, and, on the other hand, the drive current has an effect on the reluctance. The electromagnetic coupling of the HSV is exactly implemented by the permeability of the soft magnetic material in the electromagnetic force mathematical model. Therefore, the accuracy of the $B-H$ curve fitting formula directly impacts the accuracy of the results predicted by the electromagnetic mathematical model of the HSV.

Jiles and Atherton [33] proposed the Jiles-Atherton model (J-A model) to describe the phenomena of magnetization and magnetic hysteresis. The model is widely used in the electromagnetic simulation field, such as for motors. However, when applying the model, five key parameters need be determined by fitting based on test data with a complex mathematical method. Leite et al. [34] used a genetic algorithm to determine the five parameters and verified them using the least mean square error between the experimental and simulated results. Jaafar [35] studied the influence of the five parameters on the magnetization process and found that each parameter significantly impacts the prediction accuracy of the magnetization and hysteresis mathematical models. Hence, accurately determining the five parameters introduces difficulty to the engineering application of the J-A model. Therefore, a mathematical model of the $B-H$ fundamental magnetizing curve of a soft magnetic material that can be easily applied with high precision is very necessary.

Chan et al. [36] assumed that the hysteresis loop of a magnetic material is symmetrical with the $B-H$ fundamental magnetizing curve and obtained the following mathematical model for the fundamental magnetizing curve:

$$
\begin{aligned}
B_{+} & =B_{s} \cdot \frac{H+H_{c}}{\left|H+H_{c}\right|+H_{c} \cdot\left(B_{s} / B_{r}-1\right)}, \\
B_{-} & =B_{s} \cdot \frac{H-H_{c}}{\left|H-H_{c}\right|+H_{c} \cdot\left(B_{s} / B_{r}-1\right)}, \\
B & =\frac{B_{+}+B_{-}}{2} .
\end{aligned}
$$

The one-dimensional commercial software AMESim [37] used (10) to describe the $B-H$ fundamental magnetizing curve for the electromagnetic calculation, where coefficient $A$ is determined by (11):

$$
\begin{aligned}
& B=\mu_{0}\left(H+M_{s} \cdot \tanh \left(\frac{H}{A}\right)\right), \\
& A=\frac{M_{s}}{\left(B_{s} /\left(\mu_{0} \cdot H_{s}\right)\right)-1},
\end{aligned}
$$

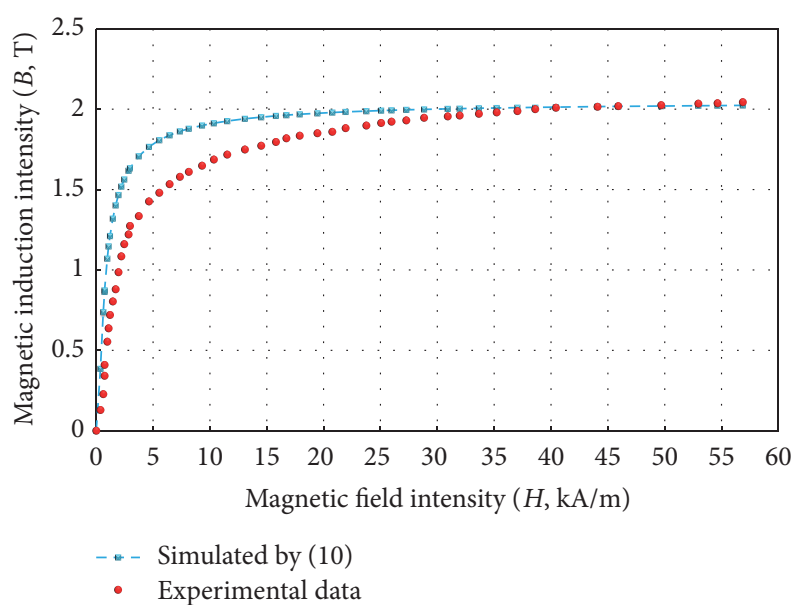

FIGURE 3: Comparison between simulated results by formula proposed by Chan and experimental results of $B-H$ curve.

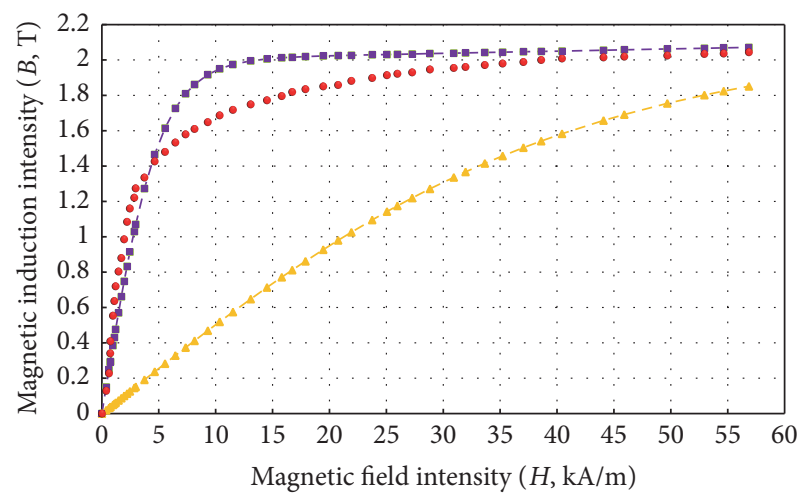

- - Simulated by (11) with $A=5000$

- Experimental data

- Simulated by (11) and (12)

FIGURE 4: Comparison between simulated results by formula proposed by AMESim and experimental results of $B-H$ curve.

where $M_{s}$ is the saturation magnetization; $B_{r}$ is the residual magnetic flux density; $B_{s}$ is the saturation induction density; $H_{s}$ is the magnetic field intensity corresponding the saturation induction density; $H_{c}$ is the coercivity; $\mu_{0}$ is the constant permeability of vacuum.

As described above, previous studies used different fitting formulas for the $B-H$ fundamental magnetizing curve; therefore, it is necessary to study the applicability of the above formula to obtain the most consistent fitting formula describing the magnetization process of a soft magnetic material. As shown in Figure 3, the equation proposed by Chan provides a good prediction at the stages of the initial magnetization and magnetic saturation but provides poor prediction in the transient process from the initial magnetization to the magnetic saturation, which is near the inflection point of the curve.

As shown in Figure 4, the results predicted by (10) and (11) cannot adequately describe the real magnetization process of magnetic materials. Equation (10) alone can provide relatively good prediction by adjusting its coefficient $A$ ( $A$ is 5000 in 


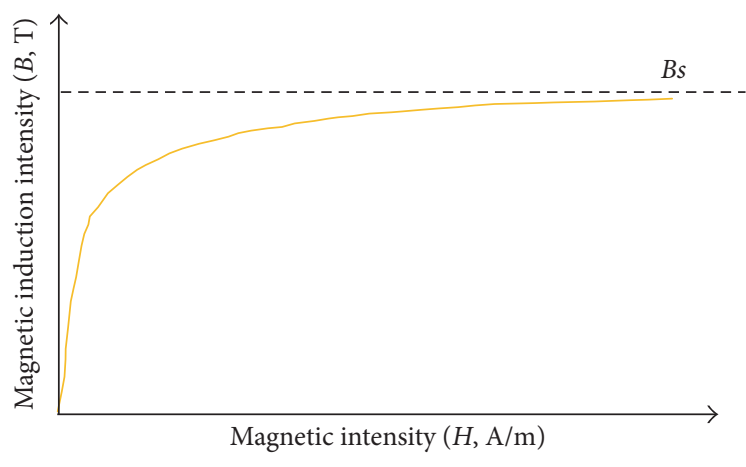

FIGURE 5: Schematic diagram of $B-H$ magnetization curve.

the prevailing circumstance), but it also provides prediction in the transient process from the initial magnetization to magnetic saturation.

Figure 5 shows the $B$ - $H$ fundamental magnetizing curve of magnetic materials, including hard magnetic materials and soft magnetic materials. With the increase of $H, B$ increases quickly at first, but its increase becomes slow and gradually approaches the saturation induction density $B_{s}$. Therefore, the selected fitting formula can accurately describe the physical phenomena of the $B-H$ fundamental magnetizing curve.

In numerous functional forms, the curve of the logarithmic function $\log (x)$ comes close to the curve in Figure 5. Additionally, when $H$ is infinite, the use of a logarithmic function can ensure that $B$ still continues to increase slightly with the further increase of $H$. This can make the permeability $\mu(\mu=\Delta B / \Delta H)$ nonzero in the whole range of $H$ and ensure that (5) and (6) have physical meaning. Intending to reduce the number of coefficients in the fitting formula of the $B-H$ fundamental magnetizing curve, the natural logarithm $\ln (x)$ is chosen as the basic functional form. However, for each type of magnetic material, the fundamental magnetizing curve starts from the coordinate origin $(0,0)$; thus, when $H$ is zero, $B$ is zero. As the natural logarithm $\ln (x)$ does not pass through this point, the equation must be changed to the form of $\ln (x+1)$; thus, the fitting formula will pass through the coordinate origin $(0,0)$. Considering the above factors, the basic functional form of the fitting formula of the $B-H$ fundamental magnetizing curve is determined as follows:

$$
B=\ln (H+1) \text {. }
$$

Every magnetic material has a different saturation induction density $B_{s}$ and maximum permeability $\mu_{\max } . B_{s}$ determines the magnetic saturation characteristics of the magnetic material, and $\mu_{\max }$ determines its nonlinear magnetization process. To ensure the universality of the proposed fitting formula, two coefficients $p 1$ and $p 2$ are introduced. $p 1$ is applied to adjust the saturation induction density $B_{s}$, and $p 2$ is applied to adjust the transformation process of the magnetizing curve from the initial magnetization to the critical magnetic saturation. Equation (13) introduces coefficient $p 1$ on the basis of (12). As shown in Figure 6, the saturation induction density of the fitting curve gradually increases with

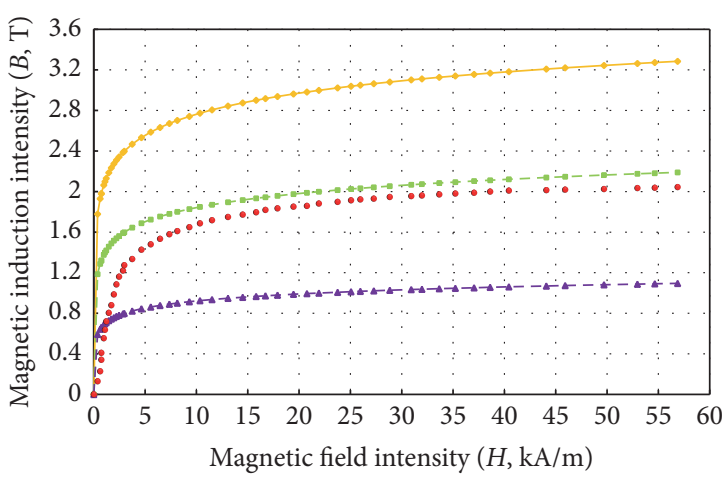

$\rightarrow$ Simulated with $p 1=0.3 \quad-\cdots$ Simulated with $p 1=0.2$ - Experimental data $\quad-\$-$ Simulated with $p 1=0.1$

Figure 6: Influence of $p 1$ on $B-H$ fitting curve.

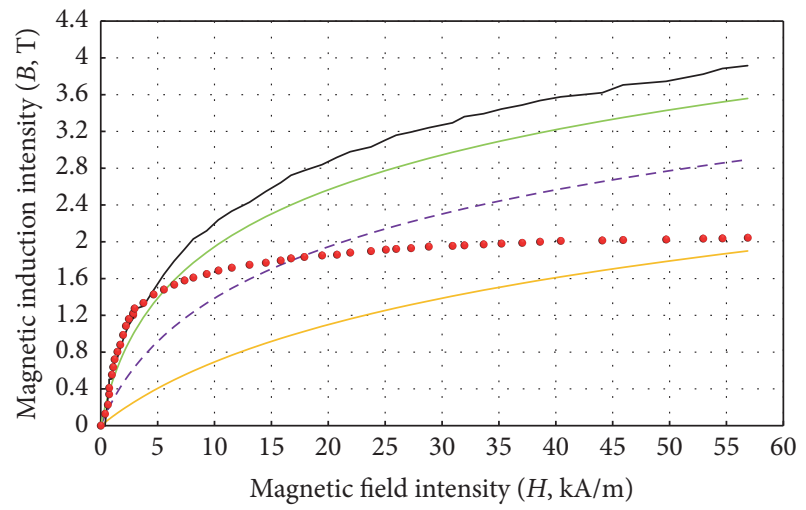

Simulated with $p 2=0.0001 \quad--$ Simulated with $p 2=0.0003$
Simulated with $p 2=0.0006 \quad-$ Simulated with $p 2=0.0009$
Experimental data

Figure 7: Influence of $p 2$ on $B$ - $H$ fitting curve.

the increase in $p 1$. When $p 1$ is 0.2 , the saturation induction densities of the simulation and experiment match especially closely. However, no matter what the value of $p 1$ is, there is a relatively large error between the simulation and experiment in the initial stage of magnetization. Hence, the equation cannot describe the nonlinear magnetization process of magnetic materials in the initial stage of magnetization:

$$
B=p 1 \cdot \ln (H+1) \text {. }
$$

Equation (14) introduces coefficient $p 2$ on the basis of (12). As shown in Figure 7, with the increase of $p 2$, the fitting curve and experimental data match more and more closely in the initial stage of magnetization. When $p 2$ is 0.0009 , (14) can particularly accurately describe the initial magnetization process of the magnetic material. However, since $p 1$ is not introduced to limit the maximum magnetic induction intensity, the predicted saturation induction density will increase with $p 2$ :

$$
B=\ln (p 2 \cdot H+1) .
$$


TABLE 1: Measurement accuracy of main equipment.

\begin{tabular}{lcc}
\hline Equipment & Force sensor & Current probe \\
\hline Type & CZLYB-3 & $1146 \mathrm{~A}$ \\
Producer & Chengdu Xingpu Transducer Co., Ltd. & Agilent Technology \\
Measurement range & $0 \sim 500 \mathrm{~N}$ & $1 \sim 100 \mathrm{~A}$ \\
Measurement accuracy & $\leq \pm 0.05 \%$ & $\leq \pm 2 \%$ \\
\hline
\end{tabular}

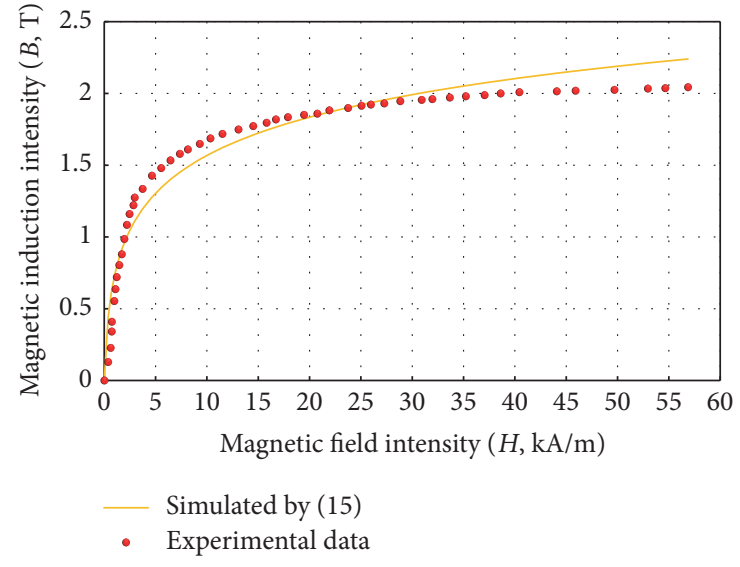

Figure 8: Comparison between simulated results by (15) and experimental results of $B-H$ curve.

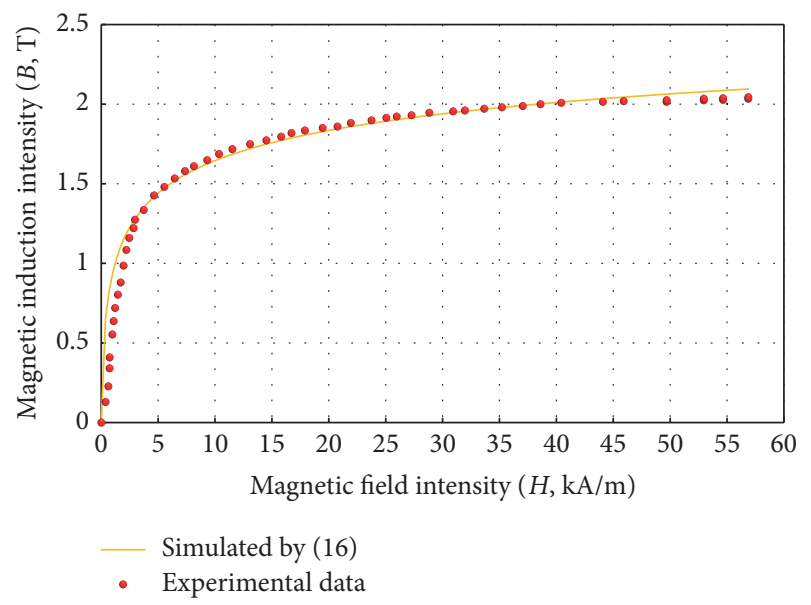

FIgURE 9: Comparison between simulated results by (16) and experimental results of $B-H$ curve.

It can be observed from the above analysis that the two coefficients $p 1$ and $p 2$ should be introduced to accurately describe the $B-H$ fundamental magnetizing curve in (12). Equation (15) introduces $p 1$ and $p 2$, which can be determined by least square method:

$$
B=p 1 \cdot \ln (p 2 \cdot H+1) .
$$

As shown in Figure 8, there is a relatively remarkable error in the magnetic saturation phase and the transition phase from the unsaturated regime to the saturated state between the simulated results from (15) and the experimental results. The reason is that the growth rate of magnetic induction intensity is relatively larger in the magnetic saturation phase. To avoid this, the square root of the logarithmic function in (15) is used. The ultimate fitting formula of the $B-H$ fundamental magnetizing curve is shown as follows:

$$
B=p 1 \cdot \sqrt{\ln (p 2 \cdot H+1)} .
$$

As shown in Figure 9, the simulated results from (16) and the experimental results match closely in the whole magnetization process, and their $R$-square determination coefficient is 0.96 . This proves that the proposed fitting formula can accurately describe not only the magnetic saturation phenomenon of magnetic materials but also the nonlinear magnetization process.

\section{Validation of Electromagnetic Model for HSV}

3.1. Test Bench of Electromagnetic Force of HSV. To experimentally validate the accuracy of the electromagnetic model for the HSV, the test bench of the static electromagnetic force depicted in Figure 10 was used. The iron core is placed at the bench free end, and the armature and force sensor (Chengdu Xingpu Transducer Co., Ltd., CZLYB-3) are positioned at the bench fixed end. The height of the free end is adjusted such that the iron core axis and the armature axis are in the same horizontal line. The total air gap is changed by adjusting the distance between the free end and the fixed end. The drive current of the solenoid is delivered by a current-controlled power amplifier, and it is measured using a current probe (Agilent Technology 1146A). The armature is attracted towards the iron core after a constant current to the coil is turned on, and the force sensor generates a weak voltage signal. This voltage signal represents the magnitude of the electromagnetic force in the axial direction after passing through the high-precision amplifier. Experimental results were obtained by changing the air gap and drive current. The measurement accuracy of the main equipment is shown in Table 1.

3.2. Model Validation. Table 2 shows the detailed structural parameters of the HSV studied in this paper. The iron core and armature are made using the same soft magnetic material whose $B-H$ fundamental magnetizing curve is shown in Figure 11. The air gap between the armature and electromagnet is adjusted to $0.1 \mathrm{~mm}$ and $0.12 \mathrm{~mm}$ in the test bed of the static characteristics of the HSV. The coil of the HSV is energized to different drive currents for the above working air gaps, and the static electromagnetic forces of the HSV at different 


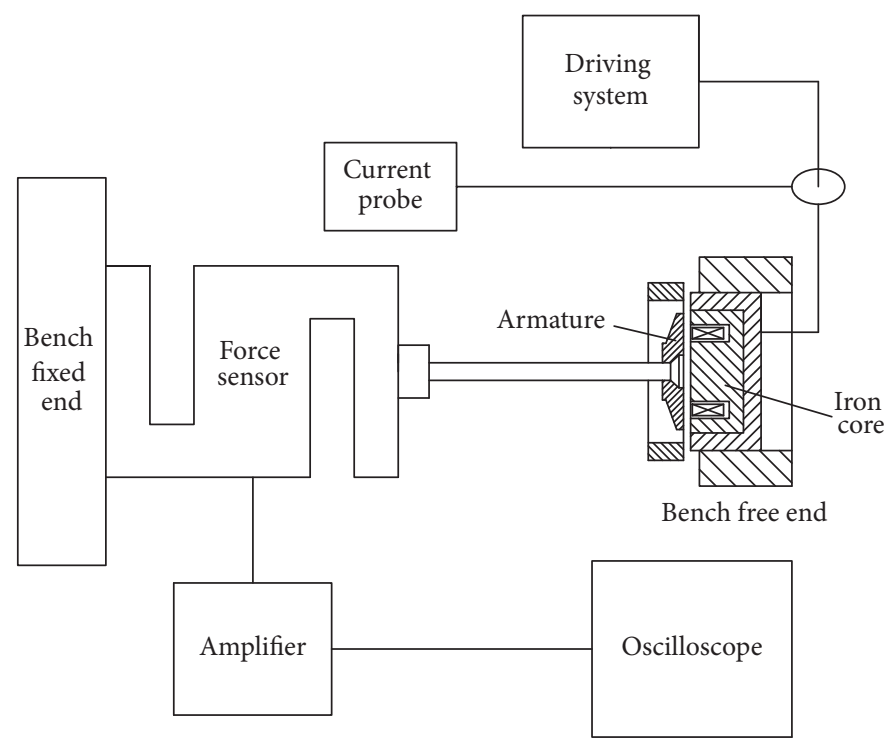

(a) The schematic block diagram of the test bench

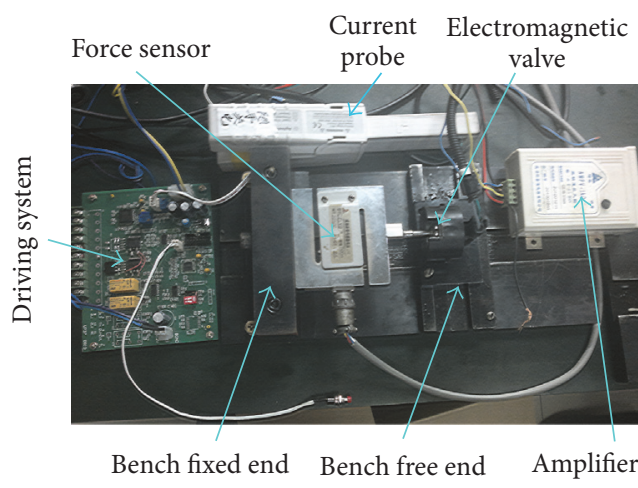

(b) Test apparatus

FIGURE 10: Test bench of electromagnetic force for HSV.

TABLE 2: Structural parameters of HSV.

\begin{tabular}{lc}
\hline Parameters & Reference value \\
\hline Iron core & \\
Height $r_{1}(\mathrm{~mm})$ & 13.7 \\
Diameter $r_{6}(\mathrm{~mm})$ & 20.4 \\
Diameter of hole $r_{3}(\mathrm{~mm})$ & 7 \\
$B$ - $H$ curve & From Figure 11 \\
Coil & \\
Number of turns $N$ & 52 \\
Height $r_{2}(\mathrm{~mm})$ & 7.6 \\
Inside diameter $r_{4}(\mathrm{~mm})$ & 12 \\
Outside diameter $r_{5}(\mathrm{~mm})$ & 17.7 \\
Armature & 1.8 \\
Thickness $r_{8}(\mathrm{~mm})$ & 20 \\
Diameter $r_{7}(\mathrm{~mm})$ & From Figure 11 \\
$B$ - $H$ curve & \\
Assembly & $0.1 ; 0.12$ \\
Working air gap $h(\mathrm{~mm})$ & \\
Control & $1 \sim 18$ \\
Driving current $I(\mathrm{~A})$ &
\end{tabular}

working air gaps and drive currents are obtained by the force sensor.

A comparison between the simulated and experimental results of the electromagnetic force at various drive currents and working air gaps is shown in Figures 12 and 13. It is easy to see that the simulated and experimental results match closely at different drive currents for the two working air gaps, and the maximum error is $15 \%$ which could meet the demands of the engineering applications. Conclusively, our developed

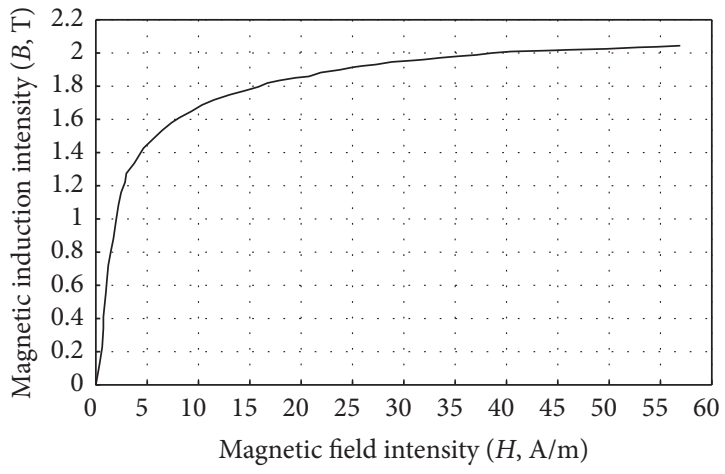

FIgURE 11: $B-H$ curve for the armature and iron core.

numerical electromagnetic model of the HSV can predict the electromagnetic force with acceptable accuracy.

\section{Results and Discussions}

As shown in Figure 14, the electromagnetic force of the HSV increases with the increasing drive current at working air gaps of $0.1 \mathrm{~mm}$ and $0.12 \mathrm{~mm}$, and it is larger with a working air gap of $0.1 \mathrm{~mm}$ than with a working air gap of $0.12 \mathrm{~mm}$ at the same drive current. This is because the electromagnetic force of the HSV is inversely proportional to the reluctance under a constant drive current and coil turns based on (1)-(4) and (9). At the same time, the smaller the working air gap is, the smaller the air gap reluctance is. Hence, the electromagnetic force at a working air gap of $0.1 \mathrm{~mm}$ is larger than that at a working air gap of $0.12 \mathrm{~mm}$ at the same drive current. The influence of the drive current on the electromagnetic force is more complex. On the one hand, the drive current affects the total magnetic flux of the HSV, and, on the other 


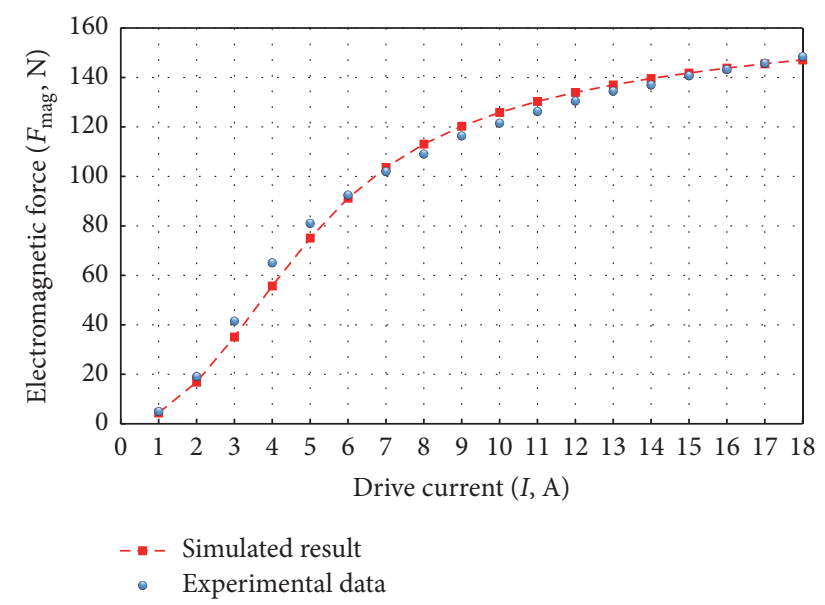

FIGURE 12: Comparison between simulated and experimental results of electromagnetic force at $h=0.1 \mathrm{~mm}$.

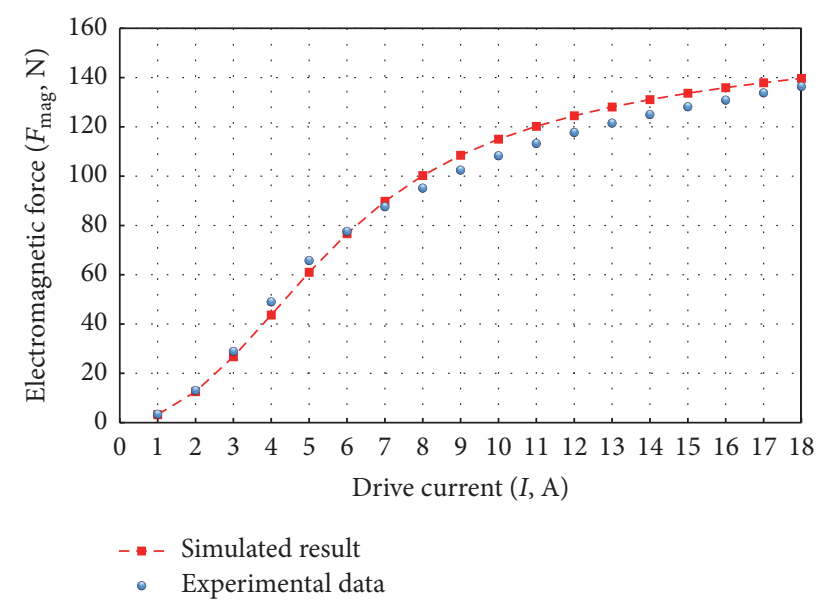

FIGURE 13: Comparison between simulated and experimental results of electromagnetic force at $h=0.12 \mathrm{~mm}$.

hand, it also affects the transient magnetic permeability of soft magnetic materials and thereby the reluctance of the iron core and armature. Therefore, to deeply analyze the influence mechanism of the drive current on the electromagnetic energy conversion of the HSV, the relationship between the increase in the electromagnetic force and the drive current is obtained by processing the test data of Figure 14 as shown in Figure 15.

It can be observed from Figure 15 that, with the increase in the drive current, the increment of the electromagnetic force increases at first and then decreases, reaching a maximum at the drive current of $4 \mathrm{~A}$. When the drive current is less than $4 \mathrm{~A}$, the increment of the electromagnetic force at a working air gap of $0.1 \mathrm{~mm}$ is always larger than that for a working air gap of $0.12 \mathrm{~mm}$; when the drive current is greater than $4 \mathrm{~A}$, the increment of the electromagnetic force is almost coincident at different working air gaps, and the electromagnetic force slowly increases as the increment gradually decreases. In fact, the change in the electromagnetic force is affected by the drive current and the total reluctance, composed of the reluctances

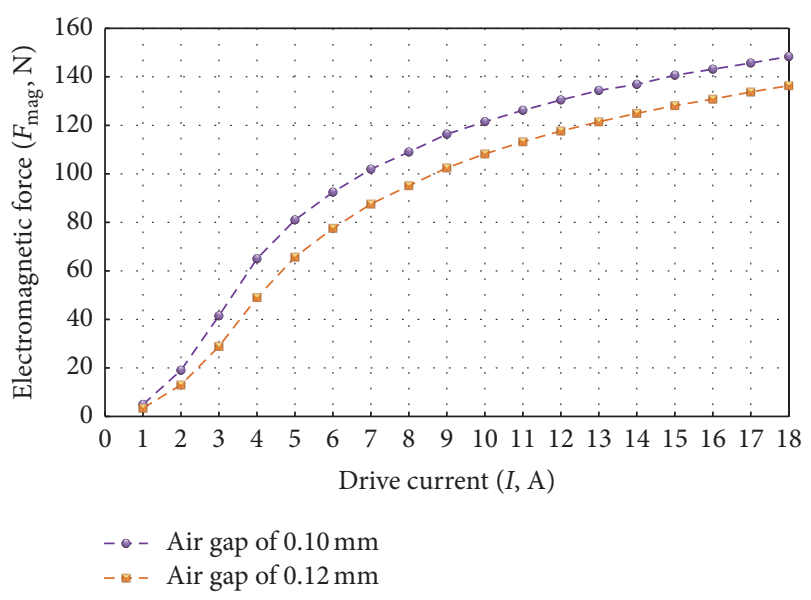

FIGURE 14: Experimental results of electromagnetic force at different working air gaps.

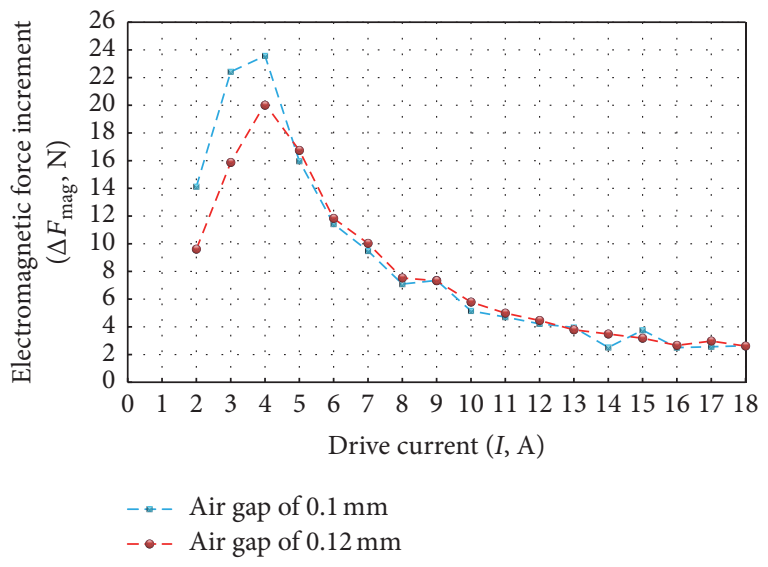

FIGURE 15: The electromagnetic force increment at different working air gaps.

of the air gap and the soft magnetic materials of the iron core and armature. The contributions of these two factors to the electromagnetic force are different within the range of different drive currents. Consequently, the angle of the variation law of the total reluctance with the drive current can better explain the variation law of Figure 15.

It can be observed from Figure 16 that the total reluctance increases with the increasing drive current at different air gaps when the drive current is less than $4 \mathrm{~A}$. However, the increase degree of the reluctance is relatively low and not enough to significantly reduce the electromagnetic force of the HSV. In this case, the increase in the drive current makes the dominant contribution to the electromagnetic force of the HSV. Accordingly, the increment of the electromagnetic force rapidly increases with the increase in the drive current at small drive current values (shown in Figure 15). When the drive current is more than $4 \mathrm{~A}$, the increase of the total reluctance is significantly influenced by the increase of the drive current, and the total reluctance increases rapidly with the increase in the drive current. Although the increase of the total magnetic flux with the increase of the drive 


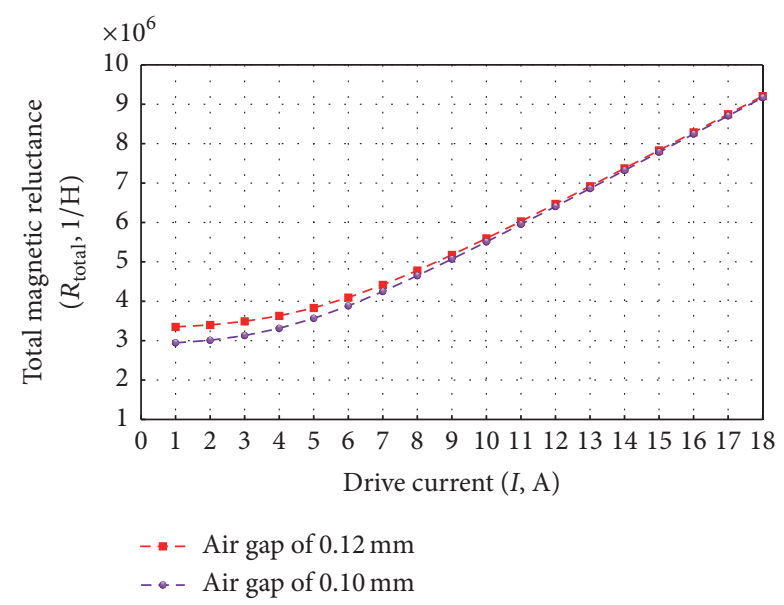

Figure 16: The total magnetic reluctance as a function of drive current at different working air gap.

current is beneficial to increasing the electromagnetic force, the contribution rate of the total reluctance to reduce the electromagnetic force is a priority. Eventually, the increment of the electromagnetic force gradually decreases.

It can be observed from Figure 16 that the total reluctance of the HSV is different at different working air gaps when the drive current is less than $4 \mathrm{~A}$, and the smaller the working air gap is, the smaller the total reluctance is. Accordingly, the electromagnetic force increment at a small working air gap is more than that at a large working air gap within a certain range of the drive current. As the drive current continues to increase, the variation curve of the total reluctance is almost coincident at different working air gaps. That is to say, the change in the total reluctance is determined by the reluctance of the soft magnetic material rather than that of the working air gap (however, it also explains that the influence of the soft magnetic material should be considered in the mathematical model of the HSV). Hence, changes in the electromagnetic force are mainly affected by the total reluctance of the HSV at the moment, according to the variation law shown in Figure 15. That is to say, when the drive current is relatively large, the electromagnetic force increment at different working air gaps has the same variation law, and the electromagnetic force increment decreases gradually.

\section{Conclusions}

(1) $B-H$ curve mathematical model easily applied with high precision is proposed and validated by the comparison of the predicted and experimental results. It can accurately describe the nonlinear magnetization and magnetic saturation phenomena in the whole magnetization process of a magnetic material, including the initial magnetization phase, the magnetic saturation phase, and the transition phase from the unsaturated regime to the saturated state.

(2) Based on the electromagnetic transient coupling principle, an electromagnetic mathematical model of the HSV is developed in Fortran language that takes the magnetic saturation characteristics into account, and it is validated by experimental data of the static electromagnetic force.

(3) Experimental research on the variation relationship between the static electromagnetic force of the HSV and its drive current at different working air gaps is carried out. It is concluded that, with the increasing drive current, the increment of the electromagnetic force first increases rapidly and then decreases at different working air gaps, and there exists a drive current value that makes the increment of the electromagnetic force achieve its maximum value.

(4) Based on the established electromagnetic mathematical model of the HSV, it is revealed that the electromagnetic energy conversion characteristics of the HSV are affected by the drive current and the total reluctance and that these two influence factors within the scope of the different drive currents have different contribution rates to the electromagnetic energy conversion efficiency.

\section{Conflicts of Interest}

The authors declare that there are no conflicts of interest regarding the publication of this article.

\section{Acknowledgments}

This work is supported by the Natural Science Foundation of Heilongjiang Province of China (LC201422), the National Natural Science Foundation of China (NSFC 51279037, 51379041, 51475100, and 51679048), the Fundamental Research Funds for the Central Universities (HEUCF160304), and Recruitment Program of High-End Foreign Experts of the State Administration of Foreign Experts Affairs (GDW20162300256).

\section{References}

[1] L. Su, X. Li, Z. Zhang, and F. Liu, "Numerical analysis on the combustion and emission characteristics of forced swirl combustion system for di diesel engines," Energy Conversion and Management, vol. 86, pp. 20-27, 2014.

[2] L. Su, X. Li, X. He, and F. Liu, "Experimental research on the diffusion flame formation and combustion performance of forced swirl combustion system for DI diesel engines," Energy Conversion and Management, vol. 106, pp. 826-834, 2015.

[3] X. Li, Z. Sun, W. Du, and R. Wei, "Research and development of Double Swirl combustion system for a di diesel engine," Combustion Science and Technology, vol. 182, no. 8, pp. 10291049, 2010.

[4] X. R. Li, H. Q. Zhou, L. W. Su, Y. L. Chen, Z. Y. Qiao, and F. S. Liu, "Combustion and emission characteristics of a lateral swirl combustion system for ID diesel engines under low excess air ratio conditions," Fuel, vol. 184, pp. 672-680, 2016.

[5] F. Luo, H. Cui, and S. Dong, “Transient measuring method for injection rate of each nozzle hole based on spray momentum flux," Fuel, vol. 125, pp. 20-29, 2014. 
[6] Z. Y. Sun, G. X. Li, Y. S. Yu, S. C. Gao, and G. X. Gao, "Numerical investigation on transient flow and cavitation characteristic within nozzle during the oil drainage process for a highpressure common-rail DI diesel engine," Energy Conversion and Management, vol. 98, pp. 507-517, 2015.

[7] Z.-Y. Sun, G.-X. Li, C. Chen, Y.-S. Yu, and G.-X. Gao, "Numerical investigation on effects of nozzle's geometric parameters on the flow and the cavitation characteristics within injector's nozzle for a high-pressure common-rail di diesel engine," Energy Conversion and Management, vol. 89, pp. 843-861, 2015.

[8] Y. Bai, L. Y. Fan, X. Z. Ma, H. L. Peng, and E. Z. Song, "Effect of injector parameters on the injection quantity of common rail injection system for diesel engines," International Journal of Automotive Technology, vol. 17, no. 4, pp. 567-579, 2016.

[9] Q. Dong, W. Long, T. Ishima, and H. Kawashima, "Spray characteristics of V-type intersecting hole nozzles for diesel engines," Fuel, vol. 104, pp. 500-507, 2013.

[10] P. Liu, L. Fan, Q. Hayat, D. Xu, X. Ma, and E. Song, "Research on Key Factors and Their Interaction Effects of Electromagnetic Force of High-Speed Solenoid Valve," Scientific World Journal, vol. 2014, Article ID 567242, 2014.

[11] Z.-Y. Sun, G.-X. Li, L. Wang, W.-H. Wang, Q.-X. Gao, and J. Wang, "Effects of structure parameters on the static electromagnetic characteristics of solenoid valve for an electronic unit pump," Energy Conversion and Management, vol. 113, pp. 119130, 2016.

[12] Q. Cheng, Z. D. Zhang, H. Guo, and N. L. Xie, "Improved processing and performance of GDI injector based on metal injection molding technology," International Journal of Applied Electromagnetics and Mechanics, vol. 44, no. 1, pp. 99-114, 2014.

[13] J. I. Miller, T. J. Flack, and D. Cebon, "Modeling the magnetic performance of a fast pneumatic brake actuator," Journal of Dynamic Systems, Measurement and Control, Transactions of the ASME, vol. 136, no. 2, Article ID 21022, 2014.

[14] Y. Shin, S. Lee, C. Choi, and J. Kim, "Shape optimization to minimize the response time of direct-acting solenoid valve," Journal of Magnetics, vol. 20, no. 2, pp. 193-200, 2015.

[15] G. M. Bianchi, S. Falfari, F. Brusiani et al., "Advanced modelling of a new diesel fast solenoid injector and comparison with experiments," SAE Technical Papers, 2004.

[16] K. F. Elmer and C. R. Gentle, "A parsimonious model for the proportional control valve," Proceedings of the Institution of Mechanical Engineers, Part C: Journal of Mechanical Engineering Science, vol. 215, no. 11, pp. 1357-1363, 2001.

[17] J. Ma, G. G. Zhu, and H. Schock, "A dynamic model of an electropneumatic valve actuator for internal combustion engines," Journal of Dynamic Systems, Measurement and Control, Transactions of the ASME, vol. 132, no. 2, pp. 1-10, 2010.

[18] A. H. Shamdani, A. H. Shamekhi, M. Ziabasharhagh, and C. Aghanajafi, "Modeling and simulation of a diesel engine common rail injector in Matlab/Simulink," in Proceedings of 14th Annual International Conference of Mechanical Engineering, Isfahan, Iran, May 2006.

[19] G. M. Bianchi, P. Pelloni, F. Filicori, and G. Vannini, "Optimization of the solenoid valve behavior in common-rail injection systems," SAE Technical Papers, 2000.

[20] Y. Wang, T. Megli, M. Haghgooie, K. S. Peterson, and A. G. Stefanopoulou, "Modeling and control of electromechanical valve actuator," SAE Technical Papers, 2002.
[21] S. K. Chung, C. R. Koch, and A. F. Lynch, "Flatness-based feedback control of an automotive solenoid valve," IEEE Transactions on Control Systems Technology, vol.15, no. 2, pp. 394-401, 2007.

[22] B. Huber and H. Ulbrich, "Modeling and experimental validation of the solenoid valve of a common rail diesel injector," $S A E$ Technical Papers, vol. 1, 2014.

[23] N.-H. Chung, B.-G. Oh, and M.-H. Sunwoo, "Modelling and injection rate estimation of common-rail injectors for direct-injection diesel engines," Proceedings of the Institution of Mechanical Engineers, Part D: Journal of Automobile Engineering, vol. 222, no. 6, pp. 1089-1101, 2008.

[24] E. E. Topçu, I. Yüksel, and Z. Kamiş, "Development of electropneumatic fast switching valve and investigation of its characteristics," Mechatronics, vol. 16, no. 6, pp. 365-378, 2006.

[25] Y. Jin, R. Deng, Y. Jin, and X. Hu, "Research on the response characteristics of solenoid valve of the air-jet loom by simulation," Journal of Thermal Science, vol. 22, no. 6, pp. 606-612, 2013.

[26] P. Naseradinmousavi and C. Nataraj, "Nonlinear mathematical modeling of butterfly valves driven by solenoid actuators," Applied Mathematical Modelling, vol. 35, no. 5, pp. 2324-2335, 2011.

[27] A. Mehmood, S. Laghrouche, and M. El Bagdouri, "Nonlinear dynamic modeling of an electro-pneumatic pressure converter for VGT pneumatic actuator," International Journal of Automotive Technology, vol. 14, no. 6, pp. 941-953, 2013.

[28] G. Sefkat, "The design optimization of the electromechanical actuator," Structural and Multidisciplinary Optimization, vol. 37, no. 6, pp. 635-644, 2009.

[29] Q. Wang, F. Yang, Q. Yang, J. Chen, and H. Guan, "Experimental analysis of new high-speed powerful digital solenoid valves," Energy Conversion and Management, vol. 52, no. 5, pp. 23092313, 2011.

[30] M. Coppo, C. Dongiovanni, and C. Negri, "Numerical analysis and experimental investigation of a common rail-type diesel injector," Journal of Engineering for Gas Turbines and Power, vol. 126, no. 4, pp. 874-885, 2004.

[31] H. Liu, H. Gu, and D. Chen, "Application of high-speed solenoid valve to the semi-active control of landing gear," Chinese Journal of Aeronautics, vol. 21, no. 3, pp. 232-240, 2008.

[32] D. T. Vu, Y. Choi, and J. Kim, "Lumped parameter modeling and analysis of hybrid magnet engine valve actuator," International Journal of Precision Engineering and Manufacturing, vol. 11, no. 6, pp. 983-986, 2010.

[33] D. C. Jiles and D. L. Atherton, "Theory of ferromagnetic hysteresis (invited)," Journal of Applied Physics, vol. 55, no. 6, pp. 2115-2120, 1984.

[34] J. V. Leite, S. L. Avila, N. J. Batistela et al., "Real coded genetic algorithm for Jiles-Atherton model parameters identification," IEEE Transactions on Magnetics, vol. 40, no. 2, pp. 888-891, 2004.

[35] M. F. Jaafar, "Magnetic hysteresis modeling and numerical simulation for ferromagnetic materials," in Proceedings of 2013 International Conference on Control, Decision and Information Technologies, CoDIT 2013, pp. 516-523, tun, May 2013.

[36] J. H. Chan, A. Vladimirescu, X. C. Gao, P. Liebmann, and J. Valainis, "Nonlinear transformer model for circuit simulation," IEEE Transactions on Computer-Aided Design of Integrated Circuits and Systems, vol. 10, no. 4, pp. 476-482, 1991.

[37] LMS Simens, “User's Guides of AMESim Rev12," 2013. 


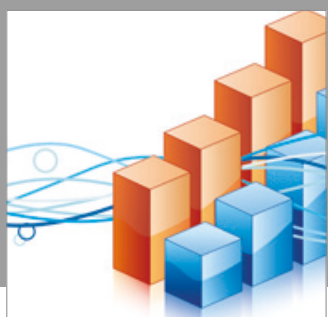

Advances in

Operations Research

vatersals

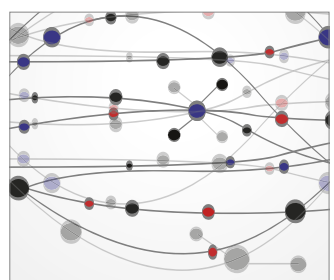

\section{The Scientific} World Journal
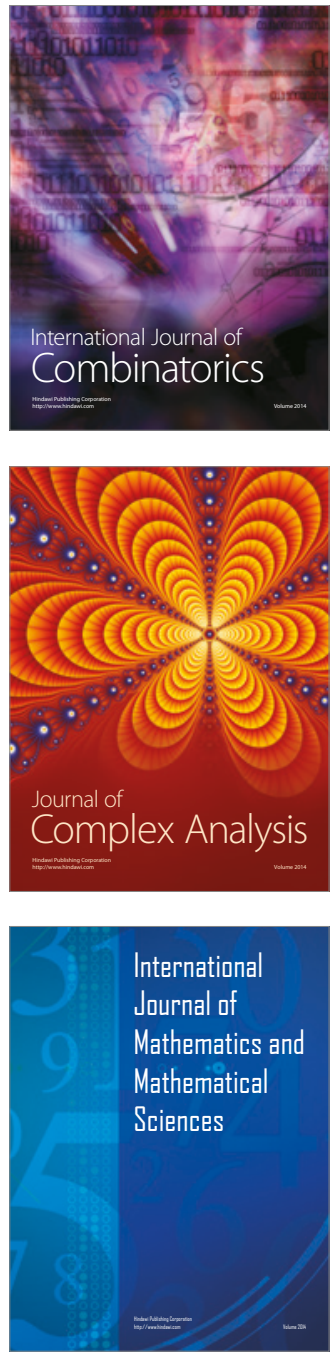
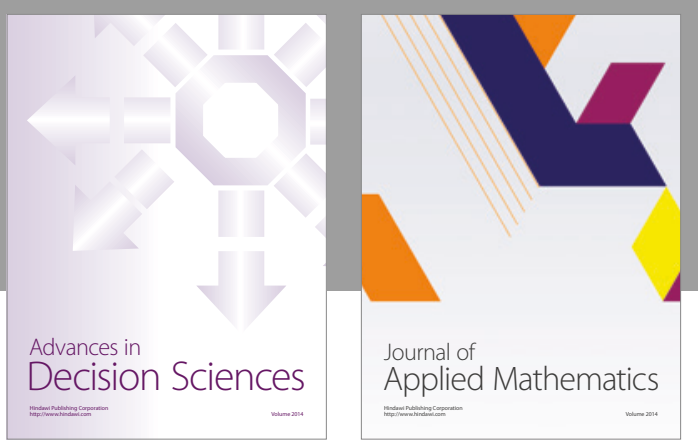

Algebra

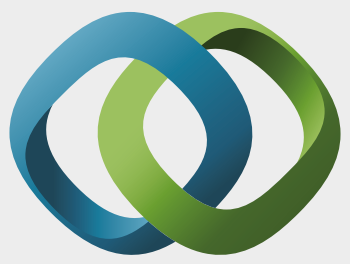

\section{Hindawi}

Submit your manuscripts at

https://www.hindawi.com
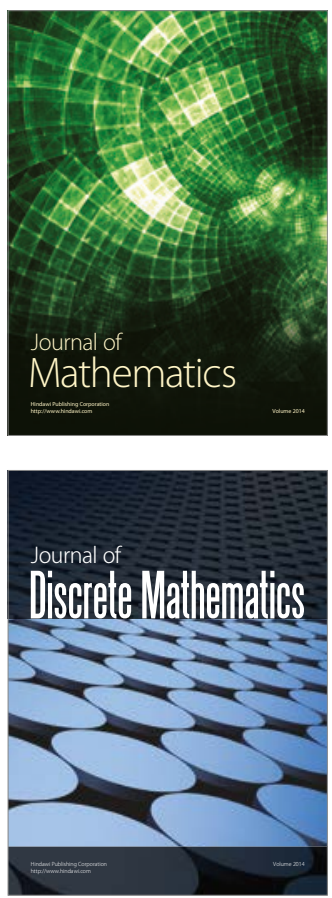

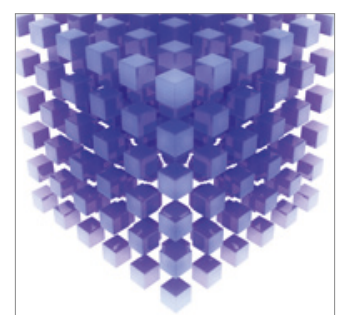

Mathematical Problems in Engineering
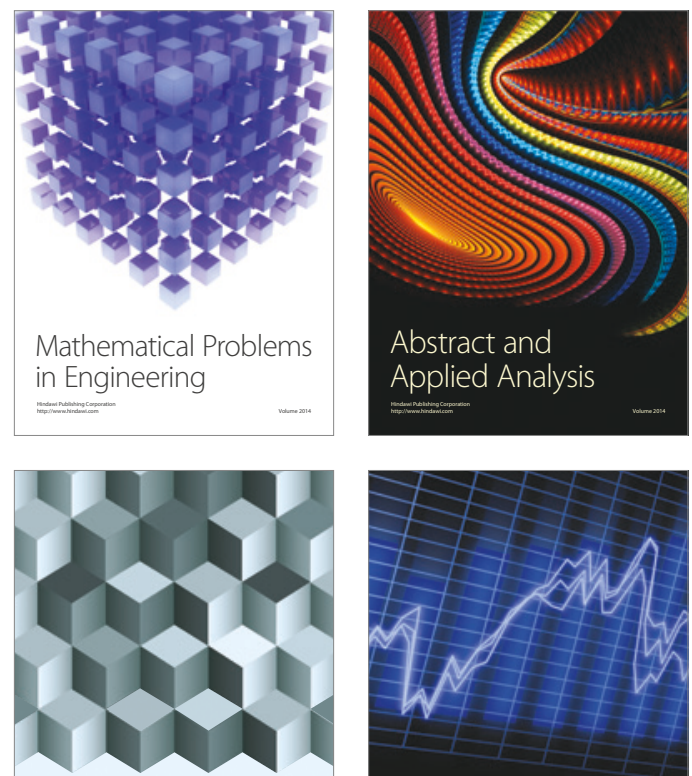

Journal of

Function Spaces

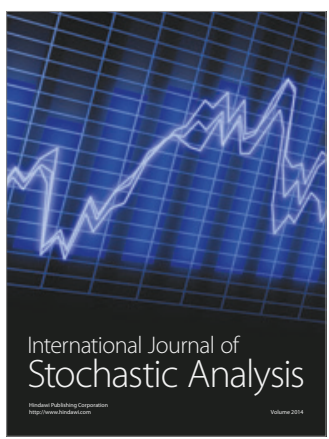

Probability and Statistics
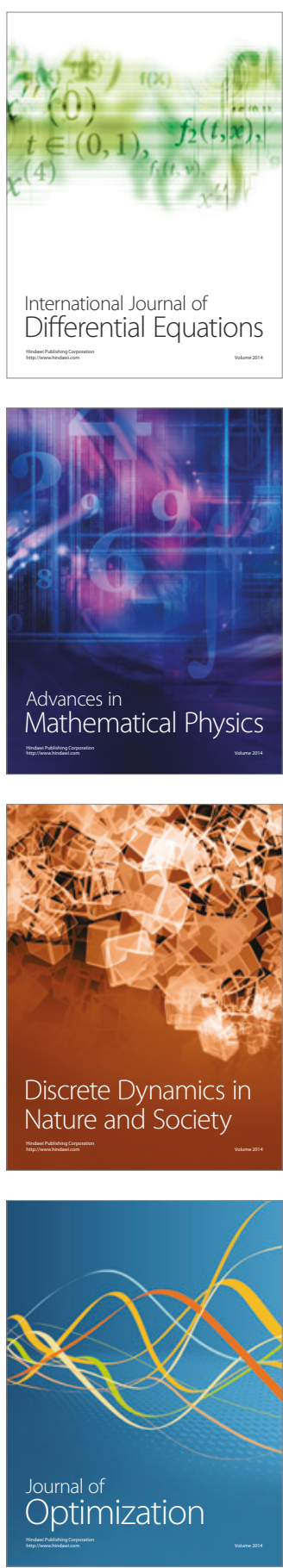